\title{
Economía y derecho en el diseño constitucional: los velos del desinterés*
}

\section{Economy and law of the constitutional design: the veil of disinterest}

Fecha de recepción: 14 de junio de 2011 Fecha de evaluación: 17 de junio de 2011 Fecha de aprobación: 21 de junio de 2011

\author{
Diego Rengifo Lozano*
}

"La razón exige que la idea, la materia y Dios, autor del perfeccionamiento de todas las cosas, sean anteriores al nacimiento del cielo".

Platón. Timeo, o De la naturaleza

\section{Resumen}

John Rawls, desde la filosofía política y del derecho, y James Buchanan, desde la economía, llegaron a perspectivas similares en términos del diseño de un orden constitucional justo. Mediante la formulación de los velos de ignorancia e incertidumbre, respectivamente en los dos autores, puede obviarse una característica aparentemente ineludible en la conducta de los individuos y que aparece como un problema fundamental en el carácter colectivo implícito en el concepto de justicia: el interés personal.

Palabras clave: Justicia, velo de ignorancia, velo de incertidumbre, economía, derecho, interés personal, norma, unanimidad, teoría de juegos, Rawls, Buchanan

\footnotetext{
* Artículo de reflexión.

** Economista y Especialista en Derecho Económico de la Universidad Nacional, Magíster en Derecho de la Universidad de los Andes, Doctorado (c) en Sociología Jurídica e Instituciones Políticas de la Universidad Externado de Colombia. Profesor de Derecho Económico, Universidad Santo Tomás.
} 


\begin{abstract}
John Rawls, with ideas from political philosophy and the philosophy of law, and James Buchanan, with ideas from economy, respectively have arrived to similar perspectives in terms of the construction of a fair Constitutional order. By means of the well-known veils of ignorance and uncertainty, both Rawls and Buchanan may allow us to get round the apparently unavoidable feature in the individual behaviour: the "personal interest", which seems to be the fundamental issue in regard to the collective character of the justice concept.
\end{abstract}

Keywords: justice, veil of ignorance, veil of uncertainty, economy, right, personal interests, rule, unanimity, games theory, Rawls, Buchanan

\title{
INTRODUCCIÓN
}

John Rawls propone un modelo de justicia en el cual los individuos eligen y determinan el contrato social con anterioridad a su vigencia. Una forma de prudencia informacional halla sustento en la elección de su estructura fundamental desde la imparcialidad, al ignorar las singulares características de la situación por ocupar con posterioridad a la firma del contrato: el velo de ignorancia. Aunque en esta construcción "la ignorancia del actor ideal se extiende únicamente a su incapacidad para identificarse con una persona particular dentro de la sociedad" (Gauthier, 1994: 311), el modelo de justicia concede a la ignorancia un valor fundamental alentando la racionalidad del sujeto, tal vez más identificable con "los otros" precisamente por la imposibilidad de hacerlo con alguno en particular: el sujeto moral kantiano, que evalúa sus acciones como si cada una de ellas tuviera la virtud suficiente para ser elevada a un principio universal de conducta. Por otro lado, James Buchanan, desde la perspectiva de la elección pública ( $p u b l i c$ choice), asume la esfera constitucional como la estructura más remota y distante del interés personal, dada su generalidad y la dificultad que supondría anticipar sus consecuencias sobre cada individuo en particular. De tal forma, advierte la posibilidad de llegar a acuerdos constitucionales consistentes y justos precisamente porque bajo esta modalidad de desconocimiento, que supondría ciertas implicaciones equivalentes al desinterés -a la que llama "velo de incertidumbre"-, resultaría viable o cuando menos más cercana la pretensión de unanimidad. El modelo resulta "justo" en tanto que ningún individuo podrá determinar cómo lo afectará una norma, es decir, no tiene seguridad en los pagos. El presente trabajo tratará de sintetizar las estructuras de los "velos" en cuanto a su convergencia con la figura del desinterés, y cómo es posible a partir de ellos lograr acuerdos políticos y constitucionales presumiblemente justos y por ende unánimes.

\section{EL VELO DE IGNORANCIA}

Ajustándonos a los términos del desconocimiento, hubiera resultado más plausible y menos notorio hallar algún tipo de valor en ese presupuesto en la Antigüedad de Sócrates, o en la Baja Edad Media de Nicolás de Cusa cuando afirmaba que "tanto más docto será cualquiera cuanto más se sepa ignorante" (Cusa, 1981, p. 24); pero no deja de llamar la atención que en los escenarios de la modernidad, donde el conocimiento es presupuesto de racionalidad, y esta última signe cualquier categoría nacida de su seno, una de sus más elaboradas criaturas pueda tomar cuerpo con 
base, precisamente, en algún tipo de "desconocimiento". Comenzaré por analizar el valor de la ignorancia en términos de justicia, aceptando cautelosamente la recomendación de Robert Nozick cuando sentencia que "ahora los filósofos políticos tienen que trabajar según la teoría de Rawls, o bien, explicar por qué no lo hacen" (1998: 183).

En su modelo, Rawls define un contrato ex-ante, donde se vería eliminado el principio gassetiano del ser en función de su circunstancia para darle cabida al kantiano del imperativo categórico.

\begin{abstract}
Se supone, entonces, que las partes no conocen ciertos tipos de hechos determinados. Ante todo, nadie conoce su lugar en la sociedad, su posición o clase social; tampoco sabe cuál será su suerte en la distribución de talentos y capacidades naturales, su inteligencia y su fuerza, etc. Igualmente, nadie conoce su propia concepción del bien, ni los detalles de su plan racional de vida, ni siquiera los rasgos particulares de su propia psicología, tales como su aversión al riesgo, o su tendencia al pesimismo o al optimismo. Más todavía supongo que las partes no conocen las circunstancias particulares de su propia sociedad. Esto es, no conocen su situación política o económica, ni el nivel de cultura y civilización que han sido capaces de alcanzar. Las personas en la posición original no tienen ninguna información respecto a qué generación pertenecen. [...] Tendrán que escoger aquellos principios con cuyas consecuencias estén dispuestas a vivir, sea cual sea la generación a la que pertenezcan (Rawls, 1997: 135).
\end{abstract}

No obstante lo racional que nos pueda parecer esta proposición objetivada a la elección de un orden justo, existen serias controversias sobre la naturaleza de la sociedad que pretende llevarlo a cabo. ¿Qué pasaría, por ejemplo, en un acuerdo ex-ante como el planteado por Rawls ante la decisión por la esclavitud o por su abolición en un escenario que, si bien me impide conocer el nivel de melanina en mi piel (antes de la convención), permite establecer que la esclavitud recae sobre la raza negra, cuya minoría se estima en el $10 \%$ de la población? ¿No podría acaso un hipotético individuo, sin menoscabo de los preceptos que exige una elección racional, precipitarse en contra de la abolición en el estado norteamericano de Georgia de mediados del XIX?

\begin{abstract}
Conceptually, the basic trouble with the maximin principle is that it violates an important continuity requirement: It is extremely irrational to make your behavior wholly dependent on some highly unlikely unfavorable contingencies regardless of how little probability you are willing to assign to them (Harsanyi, 1975: 595).
\end{abstract}

Aun atendiendo a la prescripción de Veblen, para quien "la inclinación a los juegos de azar es otro rasgo subsidiario del temperamento bárbaro" (1992: 282), debemos aceptar que si bien no sería de recibo establecer para nuestros propósitos un tipo extremo de "participante" como aquellos jugadores que la literatura recrea en las novelas de Dostoievsky, Balzac o Zweig, el hecho de situar al individuo en algún punto frente a lo desconocido conduce a leer cualquiera de sus acciones, indefectiblemente, en una dimensión de riesgo. De tal forma, para examinar estos conceptos es necesario detenernos un instante y explicar dos conceptos fundamentales que constituyen buena parte de la argumentación de los velos de ignorancia e incertidumbre, cuales son el problema de la unanimidad y la teoría de juegos.

\section{LA UNANIMIDAD COMO PROBLEMA LÓGICO}

El problema de la unanimidad y su relación con la estructura constitucional es uno de los predicamentos que se sortean con cierta solidez a partir de la instrumentalización de los velos de ignorancia e incertidumbre. Pero observemos a qué alude la dificultad en la pretensión de una- 
nimidad mediante la cual Keneth Arrow ${ }^{1}$ llegó a la conclusión de que no hay forma de deducir un agregado social, resultado de la suma de preferencias individuales, que pueda considerarse fiel a los requisitos de consistencia y que se conoce como el teorema de la Imposibilidad que lleva su mismo nombre, y a su vez se puede apreciar a partir del ejemplo original que nos proporciona la "paradoja del voto" de Condorcet:

Supongamos que existen tres individuos (A, B, C) con posibilidad de evaluar la pertinencia de tres conductas posibles (X, Y, Z) (Zuleta, 1998: 14 ss.).

A evalúa sus preferencias en el siguiente orden: $X, Y, Z$, lo cual implica que $X$ es preferible ( $>$ ) a $Y$, $Y$ es preferible $a Z, y$ por ende $X$ es preferible a $Z$.

$$
\text { A: }[X>Y ; Y>Z \text {; luego } X>Z]
$$

Simultáneamente, B organiza sus preferencias de forma diferente: $Y, Z, X$. De donde se deduce que, si bien comparte con A la preferencia de $Y$ a $Z$, disiente en la comparación de $Z$ y $X$, pues para él $Z$ es preferible a $X$ y, por tanto, de $X$ y $Y$ (una comparación compartida $y$ dos disensos con respecto a A).

$$
\text { B: }[\mathrm{Y}>\mathrm{Z} \text {; Z }>\mathrm{X} \text {; luego } \mathrm{Y}>\mathrm{X}]
$$

De igual forma, en la evaluación que realiza $\mathbf{C}$ sobre la conveniencia de las tres posibilidades, dispone el orden de sus preferencias así: Z, X, Y. Lo que implica que $Z$ es preferible $a X, y X$ es preferible a $\mathrm{Y}, \mathrm{y}$ por tanto $\mathrm{Z}$ será preferible a $\mathrm{Y}$.

$$
\text { C: }[Z>X, X>Y \text {; luego } Z>Y]
$$

Comparte con A la preferencia de $\mathrm{X}$ sobre $\mathrm{Y}$, pero disiente en lo que respecta a $Y$ y $Z$, y por ende de $Z$ y X. De otro lado, con respecto a B comparte la preferencia de $Z$ sobre $X$, pero disiente en la de Y y Z, y por tanto en la de Z y X. Una preferencia

1 Premio Nobel de Economía, 1972. compartida y dos disensos de cada uno de los individuos con respecto a los otros dos.

$$
\begin{aligned}
& \text { A: } X, Y, Z \\
& \text { B: } Y, Z, X \\
& \text { C: } Z, X, Y
\end{aligned}
$$

Procuremos establecer la "voluntad de la mayoría" respecto al orden de sus preferencias:

1. En la evaluación entre $X$ y $Y$,
a. ¿Cuántos individuos prefieren $X$ a $Y(X>Y)$ ? Dos. A y C
b. ¿Cuántos individuos prefieren $\mathrm{Y}$ a X $(\mathrm{Y}>\mathrm{X})$ ? Uno. B

Por mayoría sería necesario conceder que la conducta $\mathrm{X}$ es preferible a $\mathrm{Y}$ : $(\mathrm{X}>\mathrm{Y})$.

2. En la evaluación entre $Y$ y $Z$,
a. a) ¿Cuántos individuos prefieren $\mathrm{Y}$ a Z $(Y>Z)$ ? Dos. A y B
b. b) ¿Cuántos individuos prefieren $\mathrm{Z}$ a $Y(Z>Y)$ ? Uno. C

La norma $Y$ es preferida a la Z $(Y>Z)$.

3. En la evaluación entre $X$ y $Z$,
a. ¿Cuántos individuos prefieren $\mathrm{X}$ a Z $(X>Z)$ ? Uno. A
b. ¿Cuántos individuos prefieren $\mathrm{Z}$ a X $(\mathrm{Z}>\mathrm{X})$ ? Dos. B y C

Entonces Z es preferible a $\mathrm{X}$ : $(\mathrm{Z}>\mathrm{X})$.

De tal forma podemos observar el tipo de anarquía suscitada en la voluntad o racionalidad colectiva sobre la conveniencia o presunción de una conducta. Cómo conciliar la voluntad de los individuos con este tipo de posibilidad:

$$
\mathbf{X}>\mathrm{Y}, \mathrm{Y}>\mathrm{Z} \text { y } \mathbf{Z}>\mathrm{X}
$$

Claramente se puede apreciar que los requisitos de transitividad resultan en una suerte de imposibilidad lógica. Si 10 es mayor que 8, y 8 es mayor 
que 6 , entonces 6 no puede ser mayor que 10 . En esto consiste el gran problema de la unanimidad; en términos prácticos no sería posible establecer un norte político, social y económico con el cual todos los individuos estuvieran de acuerdo. El recurso válido que establece la democracia para conjurar este problema radica en la estructuración de las reglas del juego, el diseño de las normas.

El problema que reviste este resultado puede ser más dúctil si lo introducimos en los escenarios de las pretensiones de justicia. Dice la Corte Constitucional en la Sentencia C-651 de 1997: "La búsqueda de un ideal de justicia material consagrado en la Carta no puede confundirse con la posibilidad de que cada uno reclame la concreción de ese propósito desde su particular perspectiva y según su concepción de lo justo". Existe, pues, en términos de una lógica formal, un requerimiento de continuidad según el cual no existe posibilidad de agregar un orden de preferencias sin perjuicio de un presupuesto de consistencia, salvo en un sistema que tenga la peculiaridad de hacerse portavoz de la evaluación colectiva y cuyo dispositivo de fuerza sea, por definición, obligadamente aceptado por todos: el ámbito dictatorial que suponen las normas en términos de elección social.

Una manera interesante de leer el teorema es la de entenderlo como una demostración formal de la idea de que la sociedad es un ente que tiene existencia y fines propios sólo es compatible con un sistema de toma de decisiones sociales de índole dictatorial, donde "la razón" de la sociedad es identificada con la razón de un individuo determinado, esto es, el dictador (Zuleta, 1998: 20).

\section{EL APORTE DE LA TEORÍA DE JUEGOS Y EL CRITERIO MAXIMIN EN LA CONCEPCIÓN DE LO JUSTO}

Imaginemos el siguiente ejemplo (ver también Zuleta, 2010): dos ladrones son aprehendidos por la policía, pero esta última, pese a la convicción que tiene sobre la culpabilidad de aquellos, no posee un acervo probatorio capaz de asegurar su condena. Los sospechosos se encuentran incomunicados, lo que implica que cada uno de ellos ignora lo que pasa por la mente del otro, pero a ambos se les informa que a cada uno se le está ofreciendo un sistema de pagos (sanciones) dependiendo de la información que aporten (entiéndase, de su colaboración a la justicia). En las filas (horizontales) estableceremos las posibilidades del prisionero A (confesar o no confesar) y en las columnas (verticales) las de $B, y$ apuntaremos en cada par ordenado (A, B) los pagos a la información reportada por cada uno.

Opción I. Si ambos sindicados confiesan el delito (Sí, Sí) cada uno de ellos obtendrá un pago de cinco años de cárcel.

Opción II. Si A confiesa (Sí) y B elude su responsabilidad (No), el pago será un año de prisión para A, quien confesó, y 10 años para B.

La opción III consiste en la pareja ordenada de respuestas (No, Sí), la que acarreará un pago igual al de la opción II, un año para el que confiese y diez para el elusor, en caso de negar A y confesar B $(-10,1)$. Por último, la opción IV implica la negación del delito por ambos ladrones (No, No), con lo cual la autoridad carece de pruebas para llevar a término la acusación. De todas formas hará lo conducente para que los prisioneros cuenten con un mínimo de dos años de prisión para cada uno $(-2,-2)$, lapso que se estima apropiado para concluir la acusación por falta de pruebas, como se aprecia en la figura 1. Así, podemos expresar las opciones en la siguiente matriz de pagos:

Los términos de la utilidad de cada prisionero (jugador) se expresan en valores negativos que representan los años de condena; por tanto, los pagos reportados en la opción IV (dos años de cárcel para cada uno) sería viable, en primera instancia, sólo en el evento en que existiera la posibilidad de una negociación entre ellos que implicara un 
compromiso de silencio (no confesar). Ahora bien, aun en el evento de poder comunicarse y pactarlo, no sería razonable para ninguno de los dos cumplir el acuerdo ya que el jugador $A$, suponiendo que cumple lo pactado, corre el peligro inminente de ser traicionado por B en caso de que decida confesar, circunstancia que llevaría a este último a obtener un pago de un año de prisión y por lo que, complementariamente, A sería condenado a diez años. En ese orden de ideas, dada una hipotética negociación, no sería racional cumplirla para ninguno de los dos, ya que cualquiera de ellos que se niegue a confesar promoverá el incentivo correspondiente al otro para no cumplir el pacto de silencio. La solución del juego se establece a partir de la confesión de los dos prisioneros, cuyo pago será de cinco años de cárcel para cada uno formulada en la opción I.

Figura 1.

PRISIONERO B

\begin{tabular}{|c|c|c|}
\hline & Sí & No \\
\hline Sí & $\begin{array}{c}-5,-5 \\
\text { Opción I }\end{array}$ & $\begin{array}{c}-1,-10 \\
\text { Opción II }\end{array}$ \\
\hline No & $\begin{array}{c}-10,-1 \\
\text { Opción III }\end{array}$ & $\begin{array}{c}-2,-2 \\
\text { Opción IV }\end{array}$ \\
\hline
\end{tabular}

El individuo racional y egoísta que persigue sus propios intereses consigue unos resultados peores que si siguiera los intereses colectivos. El dilema del prisionero ha puesto en cuestión la relación entre egoísmo, racionalidad, eficiencia y justicia social. Desde el punto de vista de la eficiencia, la imposición de unas leyes desde fuera que obligaran a la cooperación aumentaría el bienestar social e individual. Esa quiebra supone la no aplicación de la teoría económica de los mercados competitivos a importantes sectores de las relaciones sociales. La eficiencia exige la cooperación y la intervención estatal porque el egoísmo y el individualismo consiguen resultados inferiores. Es decir, si se es realmente egoísta se debe cooperar (Calsamiglia, 1997: 51).
En el interior del juego existe un orden de decisiones que en el lenguaje propio de la teoría se conoce como estrategia. En nuestro ejemplo, la elección o decisión de uno de los jugadores por la opción I genera resultados superiores sin importar qué decisión tome el otro. Si uno de ellos confiesa, independiente de la estrategia que decida adoptar el otro, lo llevará a un pago superior: obtendrá cinco años de cárcel en caso de que el otro confiese, y uno solo en caso que aquel se decida por no confesar. Este tipo de estrategia, en que uno de ellos obtiene el mejor resultado posible independientemente del que obtenga el otro, y viceversa, es una estrategia dominante por cuanto se considera óptima para un jugador de manera autónoma de la que elija su adversario. Igual, una estrategia puede resultar "estable" en el evento en que ninguno de los jugadores se vea incentivado a abandonarla, so pena de salir perdiendo. Digamos que cualquiera de los dos prisioneros analiza sus pagos mínimos, cuales son diez y cinco años de prisión (uno y dos años serían los pagos máximos) y decide optar por el pago máximo entre los mínimos, lo cual corresponde a cinco años (confesar, asumiendo que el otro en su decisión le depare lo peor, es decir que también confiese). Esta estrategia de optar por el valor máximo entre los mínimos se conoce como estrategia maximin, la cual tendrá importantes implicaciones en la "posición original" de John Rawls, cuyo modelo de justicia con fundamento en la ignorancia se dirige a dos principios específicos: el primero de ellos, la libertad de cada individuo cuidada de la transgresión a la de los demás, y el segundo, el principio de diferencia, supeditado a una solución de favorecimiento al menos favorecido (maximin).

Primer principio. Cada persona ha de tener un derecho igual al más extenso sistema total de libertades básicas compatible con un sistema similar de libertad para todos.

Segundo principio. Las desigualdades económicas y sociales han de ser estructuradas de manera que sean para: 
Mayor beneficio de los menos aventajados, de acuerdo con un principio de ahorro justo, y unidos a los cargos y las funciones asequibles a todos, en condiciones de justa igualdad de oportunidades (Rawls, 1997: 280).

El mecanismo propuesto por Rawls para lograr la unanimidad sobre estos dos principios es el criterio maximin, de tal suerte que si yo no sé lo que me espera debo optar por el valor máximo entre los mínimos. Recordemos que, en el dilema del prisionero, los pagos máximos eran uno y dos años de cárcel $(-1$ y -2$)$, y los mínimos $(-10$ y -5$)$, así que haciendo uso del principio maximin debemos optar por la estrategia cuyo pago represente el valor máximo entre los mínimos. De igual forma, si ignoramos la situación que vamos a ocupar en el interior de la sociedad, debemos asumir que estaremos, en algún sentido, entre los desaventajados, y esperar que esa debilidad se vea gratificada con algún mecanismo extraordinario de amparo, o favorecimiento en palabras de Rawls. Bajo el criterio maximin, ante la ignorancia de cuál será la circunstancia que nos depare el azar, debemos optar por una distribución igualitaria, aceptando las desigualdades únicamente en beneficio de los menos favorecidos.

Podríamos recrear este planteamiento con los juegos de suma cero en los cuales una ganancia para uno de los jugadores se considera, de plano, una pérdida para el otro. Llamémosles por lo pronto juegos ordinarios. Pero existen otros que, más allá de las ganancias y pérdidas para los respectivos jugadores, son relevantes para nuestro análisis en términos de información y coordinación, como veremos más adelante.

\section{INCONVENIENTES DEL MODELO MAXIMIN}

Esta estrategia de decisión ante la incertidumbre, el criterio maximin, es refutada por John Harsanyi (1975: 595) acudiendo al siguiente juego: un hombre vive en New York City y le han ofrecido dos empleos al mismo tiempo, uno en su ciudad y el otro en Chicago. El primero es tedioso y mal remunerado, mientras el segundo es interesante y bien pagado. Digamos que decidirse por el empleo en Chicago implica un riesgo mínimo pero posible de que el avión sufra un accidente.

Figura 2.

\begin{tabular}{l|c|c|}
\multicolumn{1}{c}{ Empleo en NY } & \multicolumn{1}{c}{$\begin{array}{c}\text { Se accidenta } \\
\begin{array}{c}\text { Nendrá un pobre } \\
\text { empleo y } \\
\text { permanecerá vivo }\end{array}\end{array}$} & $\begin{array}{c}\text { Tendrá un pobre } \\
\text { empleo y } \\
\text { permanecerá vivo }\end{array}$ \\
\cline { 2 - 3 } $\begin{array}{l}\text { Empleo en } \\
\text { Chicago }\end{array}$ & $\begin{array}{c}\text { Tendrá un } \\
\text { excelente empleo } \\
\text { y permanecerá vivo }\end{array}$ \\
\cline { 2 - 3 }
\end{tabular}

Evaluar bajo el criterio maximin implicaría la condena del individuo a una situación cuyos únicos atractivos consisten en "algún" nivel de ingreso y la premisa fundamental sin la cual el ejercicio carecería de significado no tanto para nosotros como para el actor: permanecer con vida. Es necesario aquí establecer una categoría fundamental que el criterio maximin (por lo menos en este ejemplo) no introduce: el nivel de riesgo. Sea una circunstancia tal que a un individuo le dé igual (indiferente) una probabilidad del $100 \%$ de ganar 2.000 dólares $(1 \mathrm{x}$ $2.000=2.000$ ) y una probabilidad del $50 \%$ de ganar 4.000 dólares $(0,5 \times 4.000=2.000)$, decimos que es neutral al riesgo. De igual forma, se puede colegir en qué consiste una actitud proclive o aversa ante el riesgo.

El quid de la crítica de Harsanyi al criterio maximin, tal y como lo presenta Rawls en cuanto mecanismo de elección, se halla, valiéndonos de su ejemplo, en que no establece qué tan plausible es la posibilidad de accidente, ni de qué tipo de accidente se trata, ni el nivel de su predilección por un trabajo excelente y bien remunerado. Pues aun sin llegar a los extremos sobre los que hemos llamado a la precaución, nuestro jugador podría, dependiendo de esos niveles de probabilidad, elegir la opción de una vida feliz en Chicago antes que la adversidad y frustración que supone el empleo en New York. 
De lo contrario, es decir, optando por la premonición pesimista de los pagos mínimos, su contexto de libertad se vería afanosamente fragmentado:

If you took the maximin principle seriously then you could not ever cross a street (after all, you migth be hit by a car); you could never drive over a bridge (after all, it might collapse); you could never get married (after all, it might end in a disaster), etc. If anybody really acted this way he would soon end up in a mental institution (Harsanyi, 1975: 595).

En términos del análisis que nos ocupa, hemos señalado que el riesgo acompaña al azar y que este signa al jugador; por tanto, en ese entendido lo desconocido debe ser sujeto de alguna probabilidad, dado que lo único carente de ella sería la absoluta certeza. Surge entonces la inquietud sobre el porqué de la seguridad que el modelo rawlsiano impone a la consideración de que el individuo se encontrará entre los peor situados. Lejos de esta consideración, el modelo de Harsanyi asume que los presupuestos de imparcialidad pueden realizarse si el individuo se enfrenta a la misma probabilidad (equiprobabilidad) de ocupar cualquiera de las posiciones alternativas, en ningún momento la peor. Más aún, James Buchanan y Geoffrey Brennan, en La razón de las normas, llaman la atención sobre un aspecto ulterior, respecto a la seguridad de los pagos:

En la formulación de Rawls, la persona en la posición original, tras el velo de ignorancia, no sabe nada acerca de su propia posición esperada bajo las reglas del orden social elegidas, bajo los principios de justicia potencialmente operativos y seleccionados. Al mismo tiempo, sin embargo, al parecer, la persona lo sabe todo acerca de las características generales de los resultados bajo tales reglas (Buchanan y Brennan, 1985: 69).

\section{INCERTIDUMBRE VS. IGNORANCIA}

Decíamos que existe un razonamiento más allá de los juegos ordinarios donde una pérdida para un jugador se asume como una ganancia para el otro. Observemos por un momento el funcionamiento del tráfico y las reglas que lo sustentan: en ningún caso podemos afirmar que la eficiencia del sistema de normas depende de una competencia en términos de la pericia de los conductores sometidos a su imperio, sino que

[...] puede preferirse un conjunto de reglas porque hace posible la coexistencia de buenos y malos conductores en una carretera. Las reglas de tráfico tienen una función social, que es facilitar a los que utilicen la carretera la consecución de sus propósitos, con independencia del contenido de estos propósitos, y las reglas son enjuiciadas de acuerdo con su capacidad para satisfacer ese criterio (Buchanan y Brennan, 1985: 45).

La relevancia de este argumento de coordinación implícito en la norma consiste en el componente informativo que supone la obediencia a ella; en tal sentido y bajo tal supuesto, el agente accede a la posibilidad cierta de predecir lo que harán los demás.

En el siguiente juego, Buchanan describe las diferentes estrategias que resultan de considerar la norma como elemento de información y predictibilidad en escenarios de coordinación (Buchanan y Brennan, 1985: 45): un pequeño país cuyo parque automotor es prueba de la influencia inglesa y francesa, cuyos vehículos se caracterizan por el lado en el cual llevan el timón (y con ello la necesidad de conducir por la izquierda o por la derecha), debe solucionar los problemas de tráfico que resultan del cada vez mayor número de vehículos que circulan por sus calles. Esta circunstancia nos pone frente a un predicamento: establecer una norma que especifique el carril por el que se debe con- 
ducir, o bien, siguiendo la mano invisible de Adam Smith, asumir la adecuación natural y espontánea de los intereses de cada uno, como se puede apreciar en la siguiente adaptación:

Los juegos de coordinación e información se asumen de forma diferente al juego ordinario del dilema del prisionero, básicamente en virtud del contenido de predicción. Establecemos, en términos de predictibilidad, que cada una de las partes está facultada para adecuar su comportamiento en función de lo que hará la otra. En tal sentido, el modelo debe satisfacer un requerimiento de simetría. Pueden existir, en un momento dado, situaciones que generen beneficios por la decisión de optar por el no-acuerdo o, aun, por su violación. Es decir, si el ajuste es independiente, mis alternativas se adecúan a cada circunstancia en el evento de poder conducir por la izquierda o por la derecha, correspondiendo a esta "libertad" unos pagos mayores. Piénsese por un instante en un atasco vehicular en carretera.

Figura 3.

\begin{tabular}{|c|c|c|}
\hline & $\begin{array}{l}\text { Seguir la regla adoptada } \\
\text { (derecha o izquierda) }\end{array}$ & $\begin{array}{l}\text { Ajuste independiente } \\
\text { (estado de naturaleza) }\end{array}$ \\
\hline $\begin{array}{l}\text { Seguir la regla } \\
\text { adoptada } \\
\text { (derecha } \\
\text { o izquierda) }\end{array}$ & $\begin{array}{c}\text { 10, } 10 \\
\text { Opción I }\end{array}$ & $\begin{array}{c}5,11 \\
\text { Opción II }\end{array}$ \\
\hline $\begin{array}{l}\text { Ajuste } \\
\text { independiente } \\
\text { (estado de } \\
\text { naturaleza) }\end{array}$ & $\begin{array}{c}\text { 11, } 5 \\
\text { Opción III }\end{array}$ & $\begin{array}{c}-10,-10 \\
\text { Opción IV } \\
\text { Hobbe's jungle }\end{array}$ \\
\hline
\end{tabular}

Más allá de la molesta tendencia de algunos a evadir la larga fila de vehículos represados a la derecha, el comportamiento de los conductores bajo acuerdo generará de inmediato el incentivo suficiente para inducir a algún audaz conductor a sortear la demora (opción II de la figura 3). Y digo que generará el incentivo, toda vez que, en caso de no existir ningún porcentaje de conductores bajo acuerdo (opción IV), los pagos asociados al ajuste independiente general acarrearían sendos costos para la totalidad de ellos. En ese entendido, queda esclarecida la necesidad de establecer una norma (con independencia del diseño de los vehículos) y la consecuencia obvia de un consenso por parte de la totalidad de conductores. Esta unanimidad obedece al desconocimiento de los participantes sobre el carril que, en últimas, debe señalar la norma reclamada. Pero una vez agotada esa primera consideración, debemos entrar al análisis de un subjuego sobre la opción I de la figura 3, es decir prescribir por qué lado de la calle deben transitar los vehículos, a partir de consideraciones que involucren mayores especificidades en términos informativos, como lo sería la medición de agregados de vehículos dependiendo de su "certificado de origen" y otra serie de datos que supongan un mayor acervo informacional. La consecuencia directa de este incremento será la disgregación de ese primer consenso, con los consecuentes efectos sobre la pretensión de unanimidad, sobre todo en el evento en que el número de vehículos con el timón a la izquierda sea equiparable a los que lo tienen a la derecha. Digamos, pues, por simplificación, que la influencia vehicular francesa fue mayor a la inglesa y por tanto el sacrificio de los poseedores de vehículos ingleses representa un menor costo para la sociedad.

Figura 4.

Conductor $\mathrm{X}$

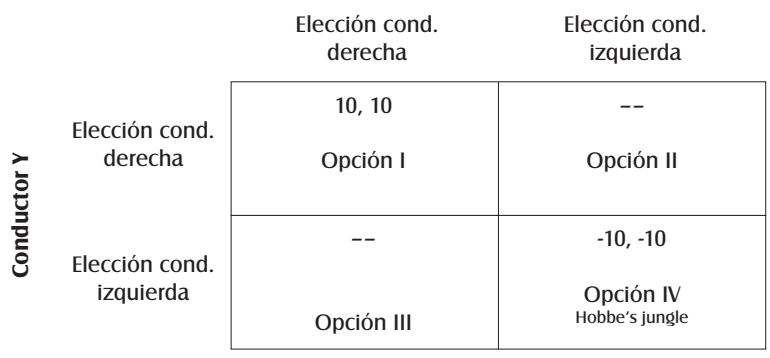

Dado que en términos de predictibilidad la regla debe dispensar información, y que esa predictibilidad debe ser de algún modo superior a los eventos y contingencias que regula (de ahí la presunción de cierto conservadurismo implícito en la norma, 
pues de otro modo esta estaría sujeta a cambios frecuentes), su vigencia como estructura funcional debe prever que un cambio en las normas produce destrucción de información, de igual forma que la decisión entre una regla o ninguna se encargó de generarla. Piénsese en el evento de una devaluación de la libra o una revaluación del franco respecto a la moneda del país en cuestión, circunstancia que podría producir un aumento inusitado en la importación de vehículos ingleses alterando los resultados que alentaron la definición de la norma evaluada en la figura 4. ¿No sería preciso revisar los contenidos que le dieron lugar respecto de los resultados pretendidos? El problema con el cambio de reglas en escenarios de coordinación se evalúa en términos de un valor esencial que se atribuye al orden como elemento predictivo.

\begin{abstract}
Si las reglas están sometidas a cambios continuos, la información que proporcionan llega a convertirse en superflua. Cada participante no puede dar por supuesto que los demás respetarán la regla existente, incluso aunque sepa lo que él personalmente va a hacer, ya que no puede saber lo que los demás sabrán que él sabe (Buchanan y Brennan, 1985: 49).
\end{abstract}

Retomando el argumento de los juegos de información y coordinación, es oportuno llamar la atención acerca de que no todas las normas, por lo menos directamente, conllevan un prurito de coordinación, pues en caso contrario, es decir si la coordinación fuera el principal pago por evaluar en las manifestaciones normativas, la ventaja de "traicionar" o evadir las normas gozaría de muy pocos adeptos. Dada una norma que genere incentivos para su incumplimiento, es decir cuando no existen pagos positivos directos que logren la asunción de la norma desde una visión cooperativa, es necesaria la adopción de pagos negativos como mecanismo que garantice su observancia. No obstante, estas sanciones se aplican a la acción, una vez se establece la norma. Pero con anterioridad a ella, el desconocimiento de los pagos asociados a la elección de la norma es lo que lleva a Buchanan a elevar la incertidumbre a una categoría sustancial del acuerdo, toda vez que los individuos desconocen cómo será afectada su circunstancia en virtud de esa elección, por lo que entre más elevada y general sea la norma, supondrá menor capacidad de cuantificación de pagos por parte del individuo.

\begin{abstract}
Nosotros estábamos operando detrás de lo que hoy llamaríamos un velo de incertidumbre; Rawls estaba operando detrás de un velo de ignorancia. Rawls decía: podemos determinar lo que es un principio de justicia para la sociedad si nos imaginamos a nosotros mismos detrás de un velo de ignorancia tal que no sabemos qué persona seremos en la sociedad, por lo cual escogeremos algo que será justo para quien sea que podamos ser. Nuestro enfoque decía: estamos analizando una regla particular que limitará los patrones de los resultados políticos. Mientras no sepamos cómo nos impacta esa regla, mientras exista esa incertidumbre, es más probable que logremos un acuerdo a nivel constitucional (Buchanan, 2001: 3).
\end{abstract}

\section{CONCLUSIÓN}

Admiramos la valentía del individuo en cuanto enfrenta resueltamente la adversidad aun a riesgo de perderse. La historia y la literatura se hallan salpicadas de este tipo de sucesos: el piquete de soldados que decide enfrentar, contra todo pronóstico favorable, una falange numerosa y bien armada, reviste un acto de valor del tipo que no lo sería si enfrentara a un enemigo debilitado y ruinoso. En este tipo de escenas la valentía se mide dependiendo de la "inseguridad de los pagos", y entre más remoto pueda parecer un desenlace favorable o más desventajosa la situación de uno de los bandos, tanto más heroico nos resultará su arrojo o, tal vez, su porfía. Bajo estos presupuestos, está de más explicar cuál de los bandos posee mayor confianza y seguridad en el resultado, cuál de ellos mayor información relevante. Extensivamente, es posible expresar los términos de justicia en el orden constitucional dependiendo de ese 
desconocimiento: la ignorancia de la situación que se va a ocupar con posterioridad al contrato es un presupuesto de justicia para Rawls, de igual forma que para Buchanan la incertidumbre sobre los pagos es un mecanismo de consenso a nivel de acuerdos constitucionales, con sus respectivas implicaciones en términos de justicia y unanimidad.

Aunque desde 1962 James Buchanan y Gordon Tullock, en El cálculo del consenso, habían configurado las bases y la estructura de desarrollo del modelo desde una formulación económica, ya desde la ilustración escocesa se pueden encontrar elementos altamente persuasivos en la explicación del orden social cifrados en mecanismos bastante cercanos a los expuestos en este escrito. Estos elementos, pese a que no pueden señalarse como cuerpos ciertos sino como meros artificios de la imaginación, explican, sin embargo, con un elevado grado de evidencia la forma en que los individuos generan el orden social sin proponérselo: la "mano invisible" y el "espectador imparcial" de Adam Smith. El concepto de orden natural, guiado por estos elementos teóricos, no se establece en los términos propios de la Investigación sobre la naturaleza y causa de la riqueza de las naciones [1776], sino que aparece diecisiete años antes en La teoría de los sentimientos morales [1759]. Más aún, el antecedente inmediato de estas categorías puede hallarse en lo que Hume definió como simpatía, que no es más que aquella cualidad de los individuos por medio de la cual convergen en sus apreciaciones.

En ese orden espontáneo existe un elemento bisagra que lleva a Hume y a Smith a la misma proposición: incorporar, a través de la simpatía, un actor fundamental en la identidad de los juicios morales: el espectador imparcial. Dice Rawls: "[...] Hume y Adam Smith suponen que si los hombres tuviesen que adoptar un punto de vista determinado, el del espectador imparcial, llegarían a convicciones similares" (Rawls, 1997: 247).
El espectador imparcial sería el elemento mediante el cual el hombre evalúa la corrección moral no sólo de actos ajenos sino, sobre todo, de los propios. La pregunta que surge es: ¿cómo conjugar el "desinterés" que puede sugerir la moral y la justicia en términos de imparcialidad y el "interés" que impone la naturaleza humana como plataforma de elección?

\begin{abstract}
Cuando nuestros sentimientos pasivos son casi siempre tan sórdidos y egoístas, ¿ cómo pueden ser nuestros principios activos frecuentemente tan nobles y desinteresados? [...] No es el apagado poder del humanitarismo, no es el tenue destello de la benevolencia que la naturaleza ha encendido en el corazón humano lo que es así capaz de contrarrestar los impulsos más poderosos del amor propio. Lo que se ejercita en tales ocasiones es un poder más fuerte, una motivación más enérgica. Es la razón, el principio, la conciencia, el habitante del pecho, el hombre interior, el ilustre juez y árbitro de nuestra conducta [...] las confusiones naturales del amor propio sólo pueden ser corregidas por la mirada de este espectador imparcial (Smith, 1997: 260).
\end{abstract}

Ante la incógnita sobre cómo conciliar los presupuestos del espectador imparcial con los requerimientos de información que, en términos propios del egoísmo, son los que definen, en últimas, el prisma con que el individuo evalúa sus acciones, dice Richard Posner: "el espectador imparcial se asemeja al hombre que se encuentra en la posición rawlsiana: en ambos conceptos se pone énfasis en que el desinterés es un elemento fundamental del concepto de justicia" (1998: 231).

\section{REFERENCIAS}

Aarnio, A. (1991). Lo racional como razonable. Madrid: Ed. Centro de Estudios Constitucionales.

Buchanan, J. (2001). Mi peregrinaje intelectual (versión electrónica). Conferencia dictada en la Universidad Francisco Marroquín (Guatemala) enero 